\title{
IMPACT OF SILICONE IMPLANT OR SALINE EXPANDER AND CONTRALATERAL SYMMETRIZATION IN QUALITY OF LIFE OF PATIENTS SUBMITTED TO IMMEDIATE RECONSTRUCTION
}

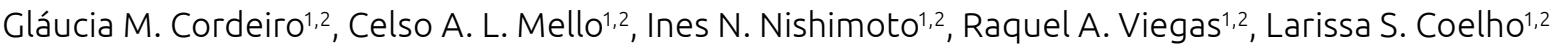
${ }^{1}$ Fundação Antônio Prudente - São Paulo (SP), Brazil.

${ }^{2}$ Hospital do Câncer Aldenora Bello - São Luís (MA), Brazil.

Objectives: Assessing Quality of Life (QoL) and aesthetic satisfaction of patients with breast cancer submitted to surgery with immediate reconstruction using silicone implant or saline expanders, with or without contralateral symmetrization. Methodology: It is a transverse study, with application of global and specific QoL questionnaires (WHOQOLBREF and BREAST-Q) in patients submitted to surgery with post-mastectomy reconstruction in Hospital do Câncer Aldenora Bello (HCAB), São Luís - MA. The nonparametric Mann-Whitney test was used to check for an association between scores. The significance level of 5\% was adopted. The patients were selected from January 2013 to May 2017, being the questionnaires applied from August to December 2017. Results: 32 patients had reconstruction and 24 (75\%) had post-mastectomy reconstruction. Eleven (45.8\%) patients were submitted to saline expander reconstruction and 13 (54.2\%) had silicone implant reconstruction. Ten (41.7\%) patients had immediate contralateral symmetrization. The analyses using BREAST-Q showed that patients with silicone implant reconstruction had greater breast satisfaction (76.5x54.4, $\mathrm{p}=0.020)$. Although it does not show statistical difference, silicone implant reconstruction tends to have better QoL on psychosocial domain (86.5x74.2, p=0.237), sexual well-being (55.5x52.5, p=0.675) and physical well-being (73.2x63.4, p=0.269). The impact of symmetrization on QoL using WHOQOL-BREEF does not have statistical significance, being greater on symmetrization group (87.5x75.9, $\mathrm{p}=0.168$ ). The analyses with BREAST-Q demonstrate that symmetrization causes better QoL related to satisfaction with breasts (79.5x57.0, $\mathrm{p}=0.022)$, psychosocial well-being (91.5x73.2, $\mathrm{p}=0.037)$, chest well-being (82.4x58.9, $\mathrm{p}=0.002)$, and quality of information $(97.0 \times 68.0, \mathrm{p}=0.007)$. Patients summited to symmetrization trends to have better sexual well-being (65.1x46.9, p=0.063). Conclusion: Patients submitted to immediate reconstruction with silicone implants presents greater breast satisfaction. Contralateral symmetrization is related to greater satisfaction with breasts, psychosocial well-being, and chest well-being. 